06

\title{
Исследование влияния размеров образцов на скорость деформации при определении прочностных динамических характеристик материала
}

\author{
(C) А.Д. Евстифеев, ${ }^{1}$ Г.А. Волков, ${ }^{1,2}$ А.А. Чеврычкина, ${ }^{1,2}$ Ю.В. Петров ${ }^{1,2}$ \\ ${ }^{1}$ Санкт-Петербургский государственный университет, \\ 199034 Санкт-Петербург, Россия \\ ${ }^{2}$ Институт проблем машиноведения РАН, \\ 199178 Санкт-Петербург, Россия \\ e-mail: Anastasiia.Che@gmail.com
}

(Поступило в Редакцию 23 января 2018 г.)

Представлены результаты тестирования аддитивного материала, изготовленного из акрилонитрилбутадиен-стирола на $3 D$-принтере. Эксперименты на динамическое растяжение выполнялись с использованием башенного копра с ускорителем. Полученные на образцах разных размеров результаты продемонстрировали возможность выполнения экспериментов с высокими скоростями деформации при растяжении за счет уменьшения рабочей части образца. Теоретический анализ полученных данных с использованием критерия инкубационного времени также показал хорошее соответствие экспериментальных данных аналитическим кривым.

DOI: $10.21883 / J T F .2019 .04 .47313 .43-18$

\section{Введение}

Технологии $3 D$-печати в последнее время находят все большее применение в повседневной жизни. И если лет десять назад они только развивались и в основном использовались для создания небольших трехмерных моделей, то сейчас они все больше внедряются в реальные производственные процессы, где производится „печать“ элементов конструкций, которые могут испытывать достаточно большие динамические нагрузки в процессе эксплуатации. Так, например, существуют проекты создания отдельных деталей автомобиля или модулей, из которых впоследствии собираются целые здания или конструкции [1]. Быстрый рост различных практических применений аддитивных технологий приводит к необходимости дополнительного изучения изменения прочностных свойств материала после этапа печати. Многие работы посвящены исследованию влияния толщины слоя [2,3], ориентации [2-4] и температуры печати [5] на механические свойства аддитивного материала. При этом с учетом потенциальных эксплуатационных условий требуется проведение экспериментальных исследований не только в случае квазистатических, но и при динамических воздействиях.

В настоящей работе приведены результаты экспериментальных исследований акрилонитрил-бутадиенстирола (ABS-пластика) в условиях статических и динамических растягивающих нагрузок. Образцы изготавливались на $3 D$-принтере двух размеров с целью оценки возможности повышения скорости деформации за счет уменьшения рабочей части. Дополнительно проведено сравнение полученных результатов с прочностными данными исходного материала [6]. Полученные экспериментальные данные были описаны с использованием структурно-временного подхода.

\section{Материал}

Испытанные образцы изготовлялись из акрилонитрилбутадиен-стирола (ABS), это ударопрочный технический термопласт, нетоксичный, устойчивый к агрессивным воздействиям. Минусом данного материала является его невысокая устойчивость к воздействию ультрафиолетовых солнечных лучей. ABS-пластик является одним из наиболее широко используемых полимерных материалов для производства различных пластиковых изделий в разных сферах: автомобилестроение, производство крупной и мелкой бытовой техники и др. Также ABS-пластик является популярным экструзионным материалом, так как его температура стеклования достаточно высокая, чтобы не возникало деформаций при небольшом нагреве, но достаточно низкая для безопасной экструзии с помощью стандартных установок.

В настоящей работе исследовались образцы, напечатанные из ABS-пластика на $3 D$-принтере. Механические характеристики образцов при скорости деформации $\dot{\varepsilon} 0.0011 / \mathrm{s}$ были определены на установке ShimadzuAG-50kNX. Было получено, что модуль Юнга $E$ равен $1700 \mathrm{MPa}$, прочность на растяжение $\sigma_{c}-40 \mathrm{MPa}$.

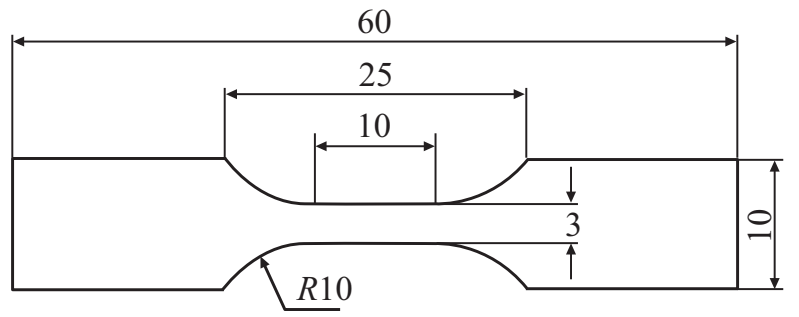

Рис. 1. Геометрические размеры образца № 1 в соответствии с ISO 8256, размеры - в миллиметрах. 

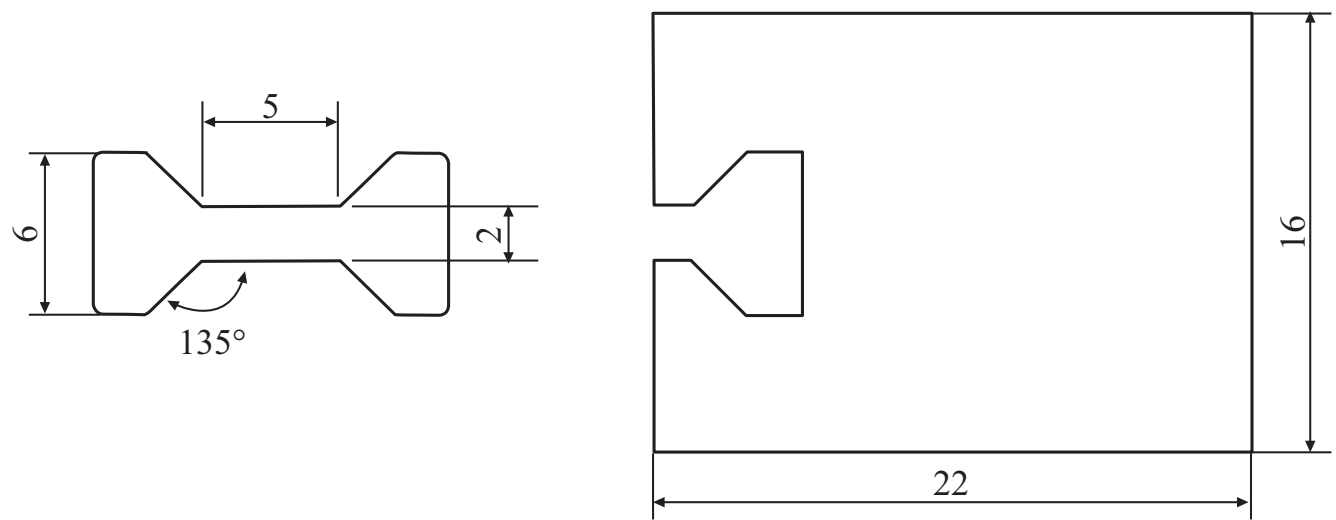

Рис. 2. Геометрические размеры образца № 2 и дополнительного захвата, размеры - в миллиметрах.

\section{Методика динамических испытаний на растяжение}

Работы по исследованию прочностных характеристик материала в условиях динамического растяжения ведутся, начиная со второй половины 20-го столетия. Наиболее распространенными экспериментальными схемами являются метод Кольского с использованием разрезного стержня Гопкинсона (РСГ) [7-9], динамические испытания по отколу $[10,11]$, сервогидравлической машины [12] и башенные копры [13]. Необходимо отметить, что в экспериментах по схеме Кольского фиксация сигналов при разрушении и их последующая интерпретация являются довольно сложной задачей. Также при обработке результатов по откольному разрушению наблюдаются достаточно большие трудности в интерпретации сигналов, полученных методами интерферометрии. Также работа на вышеупомянутых установках требует большого опыта и серьезных навыков со стороны экспериментатора. Обычно высокие скорости деформации реализуются на установках РСГ, малые и средние скорости деформации на сервогидравлических машинах и башенных копрах. В связи с этим была предпринята попытка провести высокоскоростные испытания по растяжению на наиболее простом в использовании, стандартном оборудовании, позволяющем получать скоростные зависимости прочности, для различных материалов.

В настоящей работе представлены данные высокоскоростной зависимости прочности для двух типов образцов № 1 и № 2 (рис. 1,2), которые были получены на сертифицированном оборудовании - башенном копре Instron CEAST 9350. Испытательная машина позволяет реализовать скорости деформации порядка $10^{2}-10^{3} 1 / \mathrm{s}$ для образцов № 1 .

Скорость деформации определяется, как $\dot{\varepsilon}=v / l$, где $v$ - скорость перемещения, $l$ - длина рабочей части образца. Скорость перемещения в испытаниях на растяжение на установке Instron Ceast 9350 определяется по формуле

$$
v(t)=v_{0}-\int_{0}^{t} \frac{F(s)-m g}{m} d s,
$$

где $v_{0}-$ скорость падающего груза перед ударом, которая зависит от массы падающего груза и высоты падения, $F(t)$ - регистрируемое датчиком усилие в образце в момент времени $t, m$ - масса падающего груза, $g$ - ускорение свободного падения. Правая часть в формуле (1) состоит из двух слагаемых - начальной скорости $v_{0}$ и интеграла $\int_{0}^{t} \frac{F(s)-m g}{m} d s$. Значение интеграла находится в рамках $2 \%$ от $v$ для образцов двух типов при рассмотренных режимах нагружения. Таким образом, можно принять, что скорость перемещения практически постоянна и не зависит от длины рабочей части образца. Следовательно, можно увеличить скорость деформации за счет уменьшения длины рабочей части образца при тех же внешних условиях, что и было реализовано в последующих испытаниях.

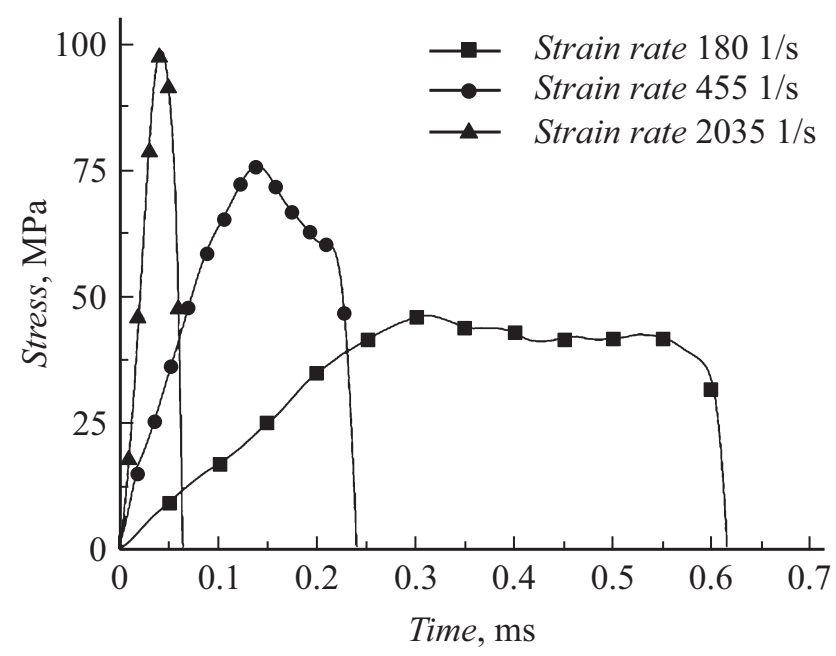

Рис. 3. Временная зависимость напряжения при растяжении образцов при различных скоростях деформирования. 
Первая серия экспериментов включала проведение испытаний на статическое и динамическое растяжение образцов № 1, размеры которых соответствовали стандарту ISO 8256 (рис. 1) [14]. В целях увеличения скорости деформации были изготовлены образцы № 2 с длиной рабочей части в 2 раза меньшей (рис. 2). Для образов № 2 были изготовлены специальные захваты (рис. 2), использование которых позволило снизить деформацию, получаемую образцами при их фиксации.

Запись данных при растяжении на копре происходит в автоматизированном режиме. На рис. 3 представлены хронограммы усилий для трех скоростей деформации. Эти данные иллюстрируют хорошее качество сигнала. Видно, что с ростом скорости приложения нагрузки увеличиваются предельные нагрузки, которые может выдержать материал. Далее будет показано, что эта зависимость между прочностью и скоростью деформации образца носит нелинейный характер и вполне закономерна.

\section{Результаты и обсуждение}

Испытания образцов из ABS-пластика выполнялись при квазистатических и динамических растягивающих нагрузках. Как уже было отмечено ранее, с ростом скорости деформации был выявлен эффект повышения предельных значений прочности. Эта особенность поведения материала хорошо описывается с использованием структурно-временного подхода, где в качестве критерия разрушения выступает критерий инкубационного времени $[15,16]$ :

$$
\frac{1}{\tau_{c}} \int_{t-\tau_{c}}^{t} \frac{\sigma(s)}{\sigma_{c}} d s \leq 1,
$$

где $\sigma$ - приложенное растягивающее напряжение, линейно возрастающее со временем, $\sigma_{c}-$ статический

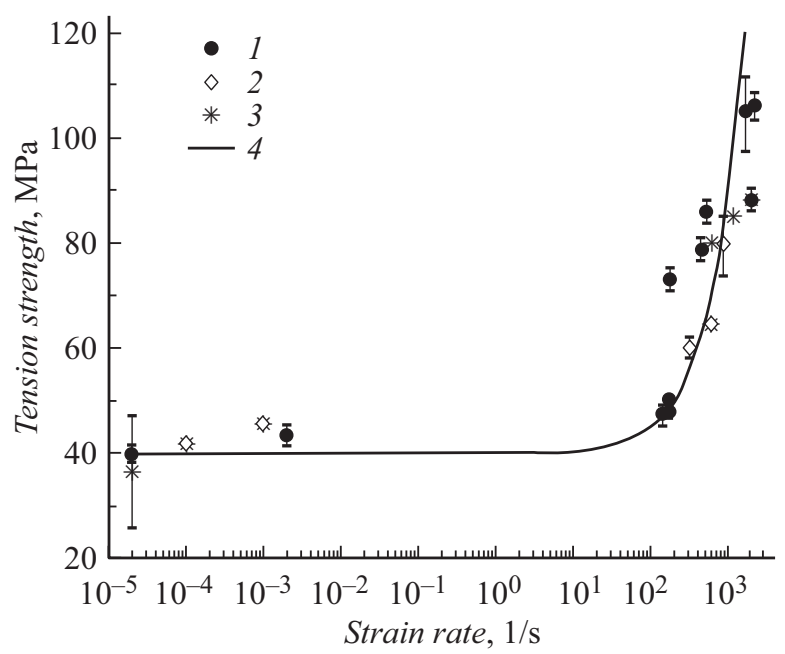

Рис. 4. Зависимость прочности на растяжение от скорости деформации для ABS-пластика: 1 - образцы по ISO 8256, 2 - уменьшенные образцы, 3 - исходный филамент [6], 4 - теоретическая кривая, построенная по критерию (2) с параметрами материала $E=1700 \mathrm{GPa}, \tau_{c}=57 \mu \mathrm{s}, \sigma_{c}=40 \mathrm{MPa}$. предел прочности при растяжении, $\tau_{c}-$ инкубационное время разрушения, отвечающее за прочность материала в условиях динамических нагрузок.

На рис. 4 представлены экспериментальные данные по двум типам образцов, напечатанным на $3 D$-принтере, и исходному материалу [6], не подвергавшемуся экструзии. В работе [6] изучалось поведение $\mathrm{ABS}$-пластика при динамическом растяжении, экспериментальное исследование проводилось на установке РСГ. Хорошее совпадение прочности аддитивного и исходного материалов обусловлено тем, что толщина слоя печати равна $0.06 \mathrm{~mm}$ и печать проводилась перекрестно под $45^{\circ}$ к продольной оси. Согласно [3], это один из способов печати, при котором прочность при растяжении получается наибольшей. Получено хорошее соответствие экспериментальных данных теоретической кривой, построенной с использованием критерия инкубационного времени (2). Определены параметры материала $E=1700 \mathrm{MPa}$, $\tau_{c}=57 \mu \mathrm{s}, \sigma_{c}=40 \mathrm{MPa}$.

\section{Заключение}

Для определения прочностных свойств ABS-материала в условиях растягивающих нагрузок были использованы разрывная машина Shimadzu AG-50kNX и башенный копер Instron CEAST 9350. Квазистатические испытания показали полное совпадение прочностных и упругих характеристик материала для двух типов образцов. При этом сравнительные динамические испытания показали, что уменьшение рабочей части образца позволяют проводить испытания при высоких скоростях деформации без использования ускорителя. Скорости порядка $10^{3} 1 / \mathrm{s}$ на „больших“ ISO 8256 достигались только при близких к предельным значениям высоты около 25 m, в то время как для уменьшенных образцов было достаточно одного метра. Это показывает принципиальную возможность увеличения скоростного диапазона испытаний на данном копре до скоростей деформаций $\sim 10^{5} 1 / \mathrm{s}$.

Работа выполнена при поддержке гранта РФФИ 18-51-80008. Раздел 3 выполнен А.А. Чеврычкиной при поддержке РНФ 17-11-01053. Экспериментальные исследования проведены с использованием оборудования ресурсного центра Научного парка СПбГУ „Исследование экстремальных состояний материалов и конструкций“.

\section{Список литературы}

[1] Lim S., Buswell R.A. et al. // Automation in Construction. 2012. Vol. 21. P. 262-268.

[2] Hart K.R., Wetzel E.D. // Engineer. Fracture Mechan. 2017. Vol. 177. P. 1-13.

[3] Ziemian S., Okwara M., Ziemian C.W. // Rapid Prototyp. Journ. 2015. Vol. 21. N 3. P. 270-278.

[4] Sood A.K., Ohdar R.K., Mahapatra S.S. // Mater. Design. 2010. Vol. 31. N 1. P. 287-295. 
[5] Rodríguez J.F., Thomas J.P., Renaud J.E. // Rapid Prototyp. Journ. 2001. Vol. 7. N 3. P. 148-158.

[6] Yin Z.N., Wang T.J. // Mater. Sci. Engineer. 2010. Vol. 527. P. 1461-1468.

[7] Owolabi G. et al. // Intern. J. Mechan. Mater. Engineer. 2016. Vol. 11. N 1. P. 3.

[8] Bragov A.M., Lomunov A.K. // Int. J. Impact Eng. 1995. Vol. 16. P. 321-330.

[9] Lindholm U.S. // J. Mechan. Phys. Sol. 1964. Vol. 12. N 5. P. 317-335.

[10] Златин Н.А., Мочалов С.М., Пугачев Г.С., Брагов А.М. // ФТТ. 1974. Т. 16. Вып. 6. С. 1752-1755.

[11] Shiro Kubota, Yuji Ogata, Yuji Wada, Ganda Simangunsong, Hideki Shimada, Kikuo Matsui // Intern. J. Rock Mechan. Mining Sci. 2008. N 45. P. 397-406.

[12] Xiao X. // Polymer Testing. 2008. Vol. 27. N 2. P. 164-178.

[13] Dean G., Read B. // Polymer Testing. 2001. Vol. 20. N 6. P. 677-683.

[14] Чеврычкина А.А., Евстифеев А.Д., Волков Г.А. // ЖТФ. 2018. Т. 88. Вып. 3. С. 392-395.

[15] Петров Ю.В. // Докл. АН СССР. 1991. Т. 321. № 1. С. $66-$ 68.

[16] Petrov Y.V., Utkin A.A. // Mater. Sci. 1989. Vol. 25. N 2. P. $153-$ 156. 